\title{
p27: tumor suppressor and oncogene ...?
}

\author{
Uta Kossatz $^{1}$, Nisar P Malek ${ }^{1}$ \\ ${ }^{1}$ Institute for Molecular Biology and Dept. of Gastroenterology, Hepatology and Endocrinology, Hannover Medical School, \\ Carl Neuberg Strasse 1, Hannover 30625, Germany.Malek.Nisar@MH-Hannover.de
}

Cell Research (2007) 17:832-833. doi: 10.1038/cr.2007.86; published online 15 October 2007

The $\mathrm{p} 27^{\mathrm{Kip} 1}$ protein was initially identified due to its ability to bind and inhibit cyclin/cdk2 complexes leading to an arrest in the G1-phase of the cell cycle. In line with the cyclin kinase inhibitory function of p27, knockout mice show a $30 \%$ increase in body size and a higher cyclin kinase activity in thymocytes leading to an accelerated passage through the cell cycle. The generation of p27 knockout mice helped to formally establish p27 function as a tumor suppressor protein. p27 knockout mice spontaneously develop pituitary tumors and treatment with carcinogenic substances like ethyl-nitrosourea or irradiation resulted in an increased mortality of the these mice as compared to wildtype or heterozygote littermates [1]. These findings confirmed the results of studies in human cancer tissues, which showed a strong correlation between low p27 expression levels and a worse prognosis of the affected patients. In contrast to other tumor suppressor proteins like $\mathrm{p} 53$ or $\mathrm{p} 16$ the genomic $\mathrm{p} 27$ locus is rarely mutated in human cancers. Its downregulation in tumors is thought to be primarily caused by an increased turnover of the wildtype protein. In fact low expression levels of p27 in human tumors frequently correlate with an increase in the expression of the pivotal F-box protein skp2 which promotes polyubiquitylation and subsequent degradation of p27 [2]. However other mechanisms especially the exclusion of p27 from the nucleus upon phosphorylation by oncogenic kinases like AKT have also been described. This mislocalization of p27 leads to the loss of its ability to inhibit kinase activity in the nucleus thereby promoting proliferation, which might help in the formation of tumors [3]. A recent paper by Besson and co-workers published in Genes and Development now challenges this conventional picture of p27 function [4].

Besson and Roberts created a mouse strain in which the wildtype version of the protein was replaced by a mu- tant form, which is unable to bind and inhibit cyclin/cdk complexes $\left(\mathrm{p} 27^{\mathrm{CK}^{-}}\right)$. As the primary function of p27 is to inhibit cyclin/cdk activity one would expect that this type of mutation should result in a phenotype comparable to what was found in $\mathrm{p} 27$ knockout mice. Remarkably though expression of the $\mathrm{p} 27^{\mathrm{CK}^{-}}$protein led to an even more pronounced tumor phenotype than what is observed in mice which have lost the protein altogether.

Comparing the incidence of lung tumors in wildtype and $\mathrm{p} 27^{\mathrm{CK}^{-} / \mathrm{CK}^{-}}$knock-in mice the authors found that loss of the ability to bind and inhibit cyclin/cdk complexes increased the lung tumor incidence from $6.25 \%$ (WT) to $26.3 \%$ (p27 ${ }^{\mathrm{CK}^{-} / \mathrm{CK}^{-}}$) while p 27 knockout mice develop lung cancers in only $2.1 \%$ of all cases. Rightfully the authors take this observation as an indication that the $\mathrm{p} 27^{\mathrm{CK}^{-}}$exerts functions which are not shared by the wildtype protein and also do not reflect the loss of the cyclin kinase inhibitory activity of p27. How then does this p27 mutant promote tumor formation and what can we learn with regard to the situation in human cancers? The idea that p27 might have activities which are beneficial for the tumor cell is not entirely new. For example in an ErbB2 dependent mouse breast cancer model, Muraoka and co-workers showed that while loss of one p27 allele accelerated breast cancer development loss of both alleles decreased cyclin D dependent kinase activity which correlated with an increased tumor latency [5]. Since the $\mathrm{p} 27^{\mathrm{CK}^{-}}$mutant is not able to bind cyclin/cdk complexes an influence on the formation of cyclin D containing complexes can be excluded as a primary cause for the tumor phenotype.

In their current work the auth ors show, that lung epihelial cells express high levels of $\mathrm{p} 27^{\mathrm{CK}^{-}}$. But while the wildtype protein is largely localized to the nucleus the $\mathrm{p} 27^{\mathrm{CK}^{-}}$ mutant is abundantly expressed in the nucleus and cytoplasm. An earlier publication by the same authors showed 
that cytoplasmic p27 is able to regulate cell motility due to p27's ability to inhibit RhoA activity [6]. Furthermore cytoplasmic expression of p27 in breast cancer cells and in Barretts associated adenocarcinoma of the esophagus correlated with a poor prognosis, indicating that cytoplasmic localization of p27 inactivates the tumor suppressor function of the protein. In addition exclusion of p27 from the nucleus is frequently found in breast cancer tissues due to an increased phosphorylation by AKT. Putting these different lines of evidence together cytoplasmic p27 would inhibit RhoA activity, which in turn would promote carcinogenesis. In fact expression of a mutant form of p27 that localizes exclusively to the cytoplasm (p27deltaNLS) downregulated RhoA and increased motility, survival, and Akt levels without an effect on cell cycle distribution in MCF7 breast cancer cells [7].

In a recent publication Jackson and co-workers showed that lung tumors can be induced by the activation of the Ras pathway [8]. However the transformation of cells by active Ras also requires activation of RhoA [9] it is therefore questionable whether the tumor promoting effect in the $\mathrm{p} 27^{\mathrm{CK}^{-} / \mathrm{CK}^{-}}$mice depends on Ras.

Importantly the tumors that arose in the $\mathrm{p} 27^{\mathrm{CK}^{-} / \mathrm{CK}^{-}}$mice originated from an excessive proliferation of a bronchioalveolar stem cell (BASC) pool. This deregulated stem cell proliferation resulted in hyperplasia and dysplasia which was followed by the development of adenomas and carcinomas. Also, adult $\mathrm{p} 27^{\mathrm{CK}^{-} / \mathrm{CK}^{-}}$mice showed a disruption of the retinal architechture with stretching of immature cells, which arose from the inner nuclear layer, into the outer layer. The comparison with the retinal phenotype of the p27 knockout mice again shows that this phenotype must be caused by a function independent of the cyclin kinase inhibitory activity of the p 27 protein. Could those data point towards a function of p27 mediated RhoA inhibition in the control of stem cell differentiation? In fact McBeath et al. recently showed that regulation of RhoA and its kinase ROCK acted as a switching mechanism to promote either adipocyte or osteoblast differentiation of mesenchymal stem cells, which were apparently regulated through changes in cell shape, a function directly affected by RhoA activity [10].

It will be important to delineate these various possibilities on a molecular level in greater detail. The generation of the $\mathrm{p} 27^{\mathrm{CK}^{-} / \mathrm{CK}^{-}}$mouse revealed an interesting new feature in the growing arsenal of p27 associated functions. While the molecular details of this mechanism remain to be elucidated the $\mathrm{p} 27^{\mathrm{CK}^{-} / \mathrm{CK}^{-}}$mouse represents an excellent model system to study the generation of malignant tumors from stem cell compartments.

\section{References}

1 Fero ML, Rivkin M, Tasch M, et al. A syndrome of multiorgan hyperplasia with features of gigantism, tumorigenesis, and female sterility in p27(Kip1)-deficient mice. Cell 1996; 85:733-744.

2 Hershko DD, Shapira M. Prognostic role of p27Kip1 deregulation in colorectal cancer. Cancer 2006; 107:668-675.

3 Viglietto G, Motti ML, Bruni P, et al. Cytoplasmic relocalization and inhibition of the cyclin-dependent kinase inhibitor p27(Kip1) by PKB/Akt-mediated phosphorylation in breast cancer. Nat Med 2002; 8:1136-1144.

4 Besson A, Hwang HC, Cicero S, et al. Discovery of an oncogenic activity in p27Kip1 that causes stem cell expansion and a multiple tumor phenotype. Genes Dev 2007; 21:1731-1746.

5 Muraoka RS, Lenferink AE, Law B, et al. ErbB2/Neu-induced, cyclin D1-dependent transformation is accelerated in p27-haploinsufficient mammary epithelial cells but impaired in p27-null cells. Mol Cell Biol 2002; 22:2204-2219.

6 Besson A, Gurian-West M, Schmidt A, Hall A, Roberts JM. p27Kip1 modulates cell migration through the regulation of RhoA activation. Genes Dev 2004; 18:862-876.

7 Wu FY, Wang SE, Sanders ME. Reduction of cytosolic p27(Kip1) inhibits cancer cell motility, survival, and tumorigenicity. Cancer Res 2006; 66:2162-2172.

8 Jackson EL, Willis N, Mercer K, et al. Analysis of lung tumor initiation and progression using conditional expression of oncogenic K-ras. Genes Dev 2001; 15:3243-3248.

9 Sahai E, Olson MF, Marshall CJ. Cross-talk between Ras and Rho signaling pathways in transformation favours proliferation and increased motility. Embo J 2001; 20:755-766.

10 McBeath R, Pirone DM, Nelson CM, Bhadriraju K, Chen CS. Cell shape, cytoskeletal tension, and RhoA regulate stem cell lineage commitment. Dev Cell 2004; 6:483-495. 